Article

\title{
Digital Pre-Distortion of Carrier Frequency Offset for Reliable Wi-Fi Enabled IoTs
}

\author{
I1-Gu Lee \\ Department of Convergence Security Engineering, Sungshin University, Seoul 02844, Korea; \\ iglee@sungshin.ac.kr or iglee19@gmail.com; Tel.: +82-2-920-7145
}

Received: 15 July 2017; Accepted: 7 August 2017; Published: 9 August 2017

\begin{abstract}
The Internet of Things (IoTs) will change the requirements for wireless connectivity significantly, mainly with regard to service coverage, data rate, and energy efficiency. Therefore, to improve robustness and reliability, WiFi-enabled IoT devices have been developed to use narrowband communication. However, narrowband transmission in WiFi such as IEEE 802.11ah causes relatively higher frequency error due to the reduced subcarrier space, which is larger than legacy wireless local area networks (WLANs) in $2.4 / 5 \mathrm{GHz}$ frequencies. In a direct conversion receiver, this error degrades the signal quality due to the presence of direct current (DC) offset cancellation circuits. In this paper, a digital carrier frequency offset (CFO) predistortion scheme is proposed for a reliable communication link in dense networks. Evaluation results demonstrate that the proposed scheme can improve received signal quality in terms of packet error rate and error vector magnitude.
\end{abstract}

Keywords: digital predistortion; carrier frequency offset; DC canceller; WLAN; narrowband Internet of Things

\section{Introduction}

Wireless local area network (WLAN) technologies are an essential feature of everyday life for the Internet of Things (IoT); the Internet of Everything (IoE) using wireless fidelity (Wi-Fi) is also beginning to emerge [1-4]. For IoT applications such as sensors and smart grid networks, WLAN technologies have been evolving to support a wide radio range and high energy efficiency by taking advantage of narrowband wireless transmission techniques for IEEE 802.11af/ah in the sub-1 GHz frequency [3-9]. However, narrowband transmission in WLAN might suffer from severe signal distortion due to the carrier frequency offset (CFO) in the reduced subcarrier space, which is relatively larger than that of legacy WLANs in $2.4 / 5 \mathrm{GHz}$ industrial scientific and medical (ISM) bands.

In this paper, for reliable communication links, digital pre-distortion of the carrier frequency offset is proposed to improve the data transmission rate by accurately estimating the CFO of a data packet transmitted through a wireless channel in a wireless communication system. The digital pre-distortion of the CFO is a method of transmitting data, channel-adaptively estimating the CFO of data packets, predistoring the $\mathrm{CFO}$ at the transmitter, and training the CFO to reduce the residual offset between $\mathrm{Tx}$ and Rx. Evaluation results demonstrate that the proposed method can minimize packet error rates by accurately estimating and compensating for a CFO in a wireless channel environment for a wireless communication system. This paper makes the following two key contributions:

- This is the first work to mitigate the effect of CFO at the transmitters for more reliable communications, improving narrowband WLANs targeted for IoT devices.

- The proposed digital predistortion of CFO yields substantial improvements in the packet error rate and residual CFO values. 
The remainder of this paper is organized as follows. In Section 2, wireless systems in narrowband are introduced. In Section 3, problematic radio frequency (RF)/analog impairments are analyzed, and related works are presented in Section 4. In Section 5, the digital CFO predistortion scheme is proposed. In Section 6, the evaluation results are presented and, finally, Section 7 draws conclusions.

\section{Wi-Fi Enabled IoTs in Narrowband}

While the IoT does not have a single and widely-accepted definition, it can be described as "things" that interact with each other in distributed wireless networks [8]. The IoT will comprise tens to hundreds of billions of heterogeneous and pervasive objects, and will enable an ecosystem with existing networks and software applications [10]. These new applications enable continuous connections with intelligent devices, and IoT solutions are increasingly becoming a staple of modern cities [11]. The IoT changes the requirements for wireless connectivity significantly, mainly with regard to its long range and need for high reliability. However, due to the proliferation of smart devices and sensors, the ISM bands for WLANs are subject to significantly higher interference than other frequency ranges [12]. Therefore, wireless connectivity technologies for IoT devices have been developed to transmit signals in narrowband.

IEEE 802.11ah supports narrower bandwidths such as 1, 2, 4, and $8 \mathrm{MHz}$. Hence, IEEE 802.11ah is considered to be more than a 10 times down-clocked version of the IEEE 802.11ac standard, allowing it to improve the receiver sensitivity $[4,13]$. These narrowband transmissions offer advantages of high noise immunity because a 10 times down-clocking of the bandwidth reduces the noise floor by $10 \mathrm{~dB}$. Furthermore, narrowband transmission is more robust to interference than is wideband transmission [14]. However, the drawback of narrowband transmission is that high frequency error on the RF crystal leads to an offset on the RF frequency. If the offset gets too big, the desired signal will be located outside the channel and be filtered out by analog low pass filters. Furthermore, the DC canceller at the receiver will filter out data subcarriers near the DC subcarrier, thus noticeably degrading the network performance and damaging the quality of service (QoS).

\section{Problematic RF Impairments in a Narrowband}

\subsection{Carrier Frequency Offset}

CFO occurs when the local oscillator frequency for down conversion in the receiver does not synchronize with the carrier frequency contained in the received signal [15]. The fundamental causes of the CFO are crystal inaccuracies and variations in the transmitter as well as the receiver side, and the Doppler effect when the mobile stations are moving. Frequency errors are typically corrected in the digital receiver side using the correlation properties of training sequences $[15,16]$. After the digital correction, very little frequency error exists. For an orthogonal frequency division multiplexing (OFDM) system, the orthogonality among subcarriers is maintained only if the receiver uses a local oscillator that is synchronous with the carrier frequency contained in the received signal [15]. Otherwise, carrier frequency mismatch between transmitter and receiver can result in inter-carrier interference (ICI).

In the IEEE 802.11 wireless LANs for $5 \mathrm{GHz}$ ISM bands, the oscillator precision tolerance is specified to be less than $20 \mathrm{ppm}$, so that the CFO is in a range from $40 \mathrm{ppm}$ to $-40 \mathrm{ppm}$ [2]. For example, if the TX oscillator is operated at $20 \mathrm{ppm}$ below the nominal frequency and if the RX oscillator is operated at $20 \mathrm{ppm}$ above, then the received baseband signal will have a carrier frequency error of $40 \mathrm{ppm}$. With a carrier frequency of $5.4 \mathrm{GHz}$ in this standard, the CFO is up to $216 \mathrm{KHz}$. In addition, if the mobile station is moving, the Doppler effect adds some hundreds of $\mathrm{Hz}$ in frequency spreading.

Even though the carrier frequency of the transmitter is usually known to a receiver, due to RF tolerance, the frequency at which the receiver operates may not exactly match the transmitter frequency. Additionally, in systems in which the transmitter or receiver is mobile, Doppler effects may also give rise to carrier frequency offsets. This offset causes a time-dependent phase shift in the received signal after conversion to the baseband, which can cause errors in the reconstruction of the transmitted signal. 


\subsection{Offset}

DC offset arises from nonlinearity and self-mixing in the front-end [8,14]. Large DC offset at the output of the mixer is amplified by large gain and causes saturation of the following RX path. When this happens, the DC offset will cause the receiver to fail. Typically, DC offset cancellation circuits can be included in the signal path in order to remove DC components. For typical WLAN direct conversion receivers, most DC offsets have to be mitigated in the analog domain to prevent clipping signals due to large DC offsets at high gain settings; then, a digital DC canceller compensates for the residual DC offset. Popular DC offset compensation schemes include offset estimation/cancelling, AC-coupling, and many other methods that use adaptive techniques [8].

However, if the high pass filter (HPF) pole is placed too high in frequency domain, it may cause a severe degradation in the error vector magnitude (EVM) of the lower subcarriers. At the same time, the filter poles cannot be placed too low in frequency because this would result in a very slow transient response. Direct conversion receivers reduce the circuit complexity and power consumption of superheterodyne receivers. Direct conversion receivers, however, are susceptible to noise from sources including DC offset and carrier frequency offset.

\subsection{Combining Effects of CFO and DC Offset}

Typically, in OFDM systems, no data is transmitted on the DC subcarrier. However, injection of DC components may occur at the receiver due to impairments. OFDM systems require accurate frequency synchronization between the receiver and the transmitter because, with frequency deviation, the sub-carriers will not be orthogonal, potentially resulting in inter-carrier interference. The injection of such a DC component in conjunction with any uncertainty in the CFO may limit the performance of the communication system. The samples of the signal received by the antenna may be represented by the following formula:

$$
Y[n]=x[n] e^{j(\omega+\delta \omega) n}+D+z[n] .
$$

$D$ is the DC offset introduced at the receiver. $\omega+\delta \omega$ is the normalized (by sampling frequency) radian carrier frequency offset (CFO), where $\omega$ is the CFO estimated by the receiver and $\delta \omega$ is the residual unknown CFO. $x[n]$ represents the complex-valued time-domain baseband samples of the received waveform, and $z[n]$ identifies additive noise samples. Alone, the DC offset introduced at the receiver does not have a large detrimental effect on the receiver performance. However, the combination of the DC offset and the addition of any uncertainty as to the carrier frequency offset, when the exact transmission frequency is unknown, to the receiver tends to cause significant adverse effects in receiver functionality. To combat the distortive effects of the combination of an uncertain CFO and a high DC offset, a high pass DC component filter may be added to the receiver. The high pass filter minimizes any DC offset that may be introduced by the receiver. In the absence of CFO, DC offset can be measured and corrected in the digital domain using a training sequence included in the transmitted signal. For instance, the IEEE 802.11a standard for wireless communication provides for long and short training sequences to be included in the transmitted data. These training sequences are made up of symbols that do not include any DC component; in the absence of DC offset, the average of the samples over a period of one training symbol would be zero. Thus, averaging of received samples corresponding to symbols of either training sequence can be used to estimate DC offset. However, the CFO introduces a time dependent phase shift that causes the average of the samples over the training sequence period to be non-zero even in the absence of DC offset, rendering this technique unreliable. It would therefore be desirable to provide a more reliable technique for estimating and correcting a DC offset in the digital domain in the presence of CFO.

\section{Related Work}

Many studies have been done to provide reliable communication links to users in IEEE 802.11 standards [15-18]. In the conventional wireless devices, a CFO is estimated by a unit of a packet 
using a preamble located at a header of a data packet transmitted through a wireless channel in an OFDM wireless communication system $[17,18]$. The conventional wireless systems compensate the $\mathrm{CFO}$ by estimating the $\mathrm{CFO}$ based on the received frames at the receiver. Furthermore, data is restored by compensating for the estimated CFO when a signal field and a data field of the data packet are restored. An OFDM communication system transmits and receives a massive amount of data through multi-subcarriers. In the OFDM communication system, each subcarrier of a wireless channel has an orthogonal property that transmits and receives multi-subcarriers. In order to improve the data transmission rate of a limited wireless channel, the OFDM communication system determines the channel state of a wireless channel and controls the transmitting and receiving of data based on the determined channel state of the wireless channel.

The OFDM communication system estimates a CFO using a unit of a packet; it then restores the transmitted data packet by compensating for the estimated CFO. However, it is difficult to accurately estimate a CFO when the wireless channel state is very poor due to noise, interference or channel effects, for example when the signal to interference and noise ratio (SINR) of a wireless channel is smaller than a predetermined threshold value. When the transmitted data packet is restored by compensating for an inaccurately compensated CFO, the packet error rate increases and throughput drops. Particularly, if the mobile terminal in the OFDM communication system is in a dense network, channel variation and interference are further and more seriously generated and the wireless channel state abruptly varies. Accordingly, inaccurate estimation of the CFO and the data packet error are further and frequently generated. As a result, the data transmission rate significantly deteriorates and overall system performance also rapidly drops.

Therefore, there have been demands for the development of a data transmitting/receiving method to minimize errors generated while restoring data packets and improving data transmission rate by accurately estimating the $\mathrm{CFO}$ of data packets transmitted through wireless channels in wireless communication systems such as the OFDM communication system. When a CFO estimation error is generated due to a poor wireless channel state such as low SINR in a dense network, an error is generated while restoring the information included in a signal field, such as the data packet length and the data transmit rate. Accordingly, an error is generated in the next data packet or data packet transmission is delayed because the end of the data packet is determined inaccurately. As a result, system performance deteriorates.

\section{Digital Pre-Distortion of CFO}

Using preambles, a receiver can estimate the link quality such as the signal-to-interference noise ratio (SINR). The long training field is 8 us in length, and is composed of two identical 3.2 us symbols. Due to the symbol repetition, this long training field can be used to estimate SINR [19]. In order to improve the data rate, the $\mathrm{CFO}$ values of data packets transmitted through wireless channels can be estimated by the units of packets in the physical layer (PHY); then, the estimated CFO and an average value of the CFOs estimated from the packets in good SINR conditions are stored, an estimated CFO of a current data packet is compensated for, or the average CFO is compensated according to the wireless channel environment. The transmitter distorts and sends a data packet as much as the CFO, which is estimated at the receiver. Accordingly, the receiver can further reliably detect and decode the packets. As a result, the data rate is improved by minimizing the residual CFO.

Figure 1 provides a flowchart and an algorithm illustrating the method of the proposed digital CFO predistortion. If a measured long-term SINR is larger than a predetermined threshold value (Threshold 1), it is quickly adapted to channel variation because the CFO is estimated and predistorted by the unit of the packet. If the long-term SINR variation is larger than a predetermined threshold value (Threshold 2), a short-term SINR is monitored to determine if the stored CFO is used or not. When the short-term SINR is smaller than Threshold 1, it is difficult to accurately estimate the CFOs of the data packets transmitted through the wireless channel. That is, the estimated error may be very significant. In this case, the data packets are restored by compensating for an average CFO of the CFOs 
estimated in good SINR conditions. If the short-term SINR is larger than Threshold 1, the transmitter predistorts the $\mathrm{CFO}$ using the currently estimated $\mathrm{CFO}$.

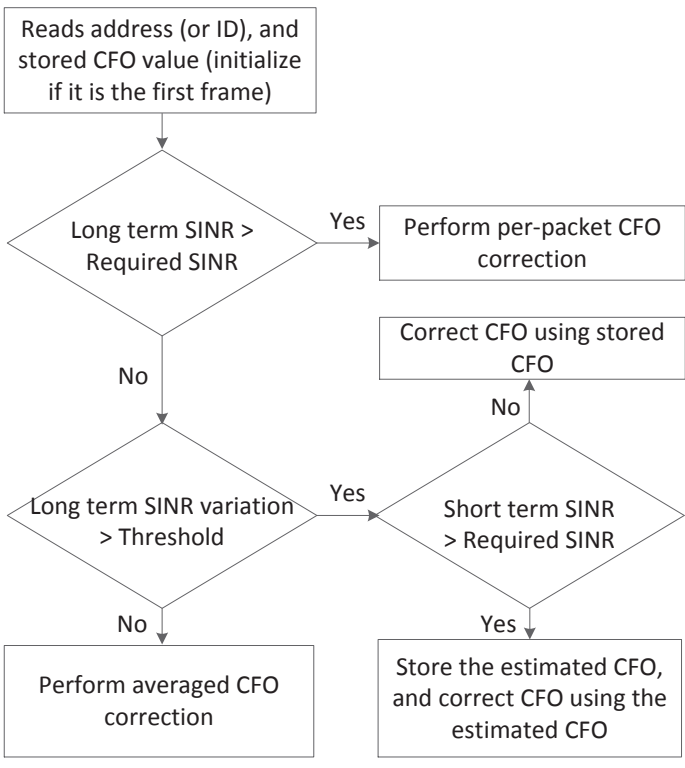

(a)

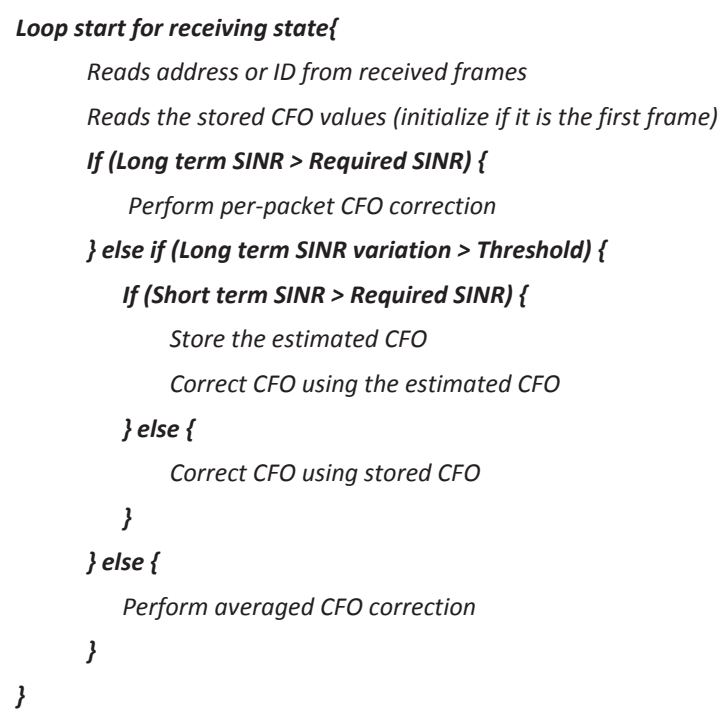

(b)

Figure 1. (a) flowchart and (b) algorithm for digital carrier frequency offset (CFO) predistortion.

As described above, the compensated CFO, which is compensated restoring a previously received packet, is distorted in advance for the next transmission packet. That is, in a communication environment, a transmitter transmits packets by distorting the compensated CFO in advance. Accordingly, a receiver receiving packets can accurately detect and decode packets. As a result, data can be stably and reliably transmitted and received. The channel state of a wireless channel is estimated when the data packet is transmitted through the wireless channel. That is, the SINR of the wireless channel is estimated, the estimated SINR is compared with a predetermined first threshold, and the channel state of the wireless channel is estimated based on the comparison result. When it is determined that the current link quality is worse than the link quality required to estimate the CFO, the current data packet is restored by compensating the estimated CFO of the current data packet to the estimated CFO of the previously transmitted data packet. When it is determined that the current link quality is better than the required link quality, the current data packet is restored by compensating the estimated CFO of the current data packet to the estimated CFO of the current data packet.

Accordingly, packet restoration error and packet loss can be prevented by accurately estimating the CFO without failing to estimate the CFO of the data packet, which may occur when a wireless channel has a poor state such as that of a wireless channel having a small SINR, multipath fading, or large delay. Therefore, the data packet transmit rate, the data processing rate, and the coverage thereof are improved, and overall system performance is improved.

\section{Performance Evaluation}

In this section, the evaluation methodology and results are presented. The proposed digital pre-distortion (DPD) scheme for CFO correction was evaluated using a computer simulation. To evaluate the performance of the proposed scheme, the simulation model was built on a $C$ simulator with the IEEE 802.11 reference channel model [20].

Figure 2 shows signal analysis results, which indicate fairly significant impairments of the lower index subcarriers. This can arise from excessive $\mathrm{CFO}$ and as a result of cutting off the low frequency subcarriers by use of a high pass filter with too high of a corner frequency. Figure 3 shows the signal 
qualities over the CFOs in additive white Gaussian noise (AWGN) and Fading channel. The average SINR was $30 \mathrm{~dB}$ for both channel conditions; there was a $50 \mathrm{~ns}$ root mean square (RMS) delay spread channel condition in the Fading channel condition.

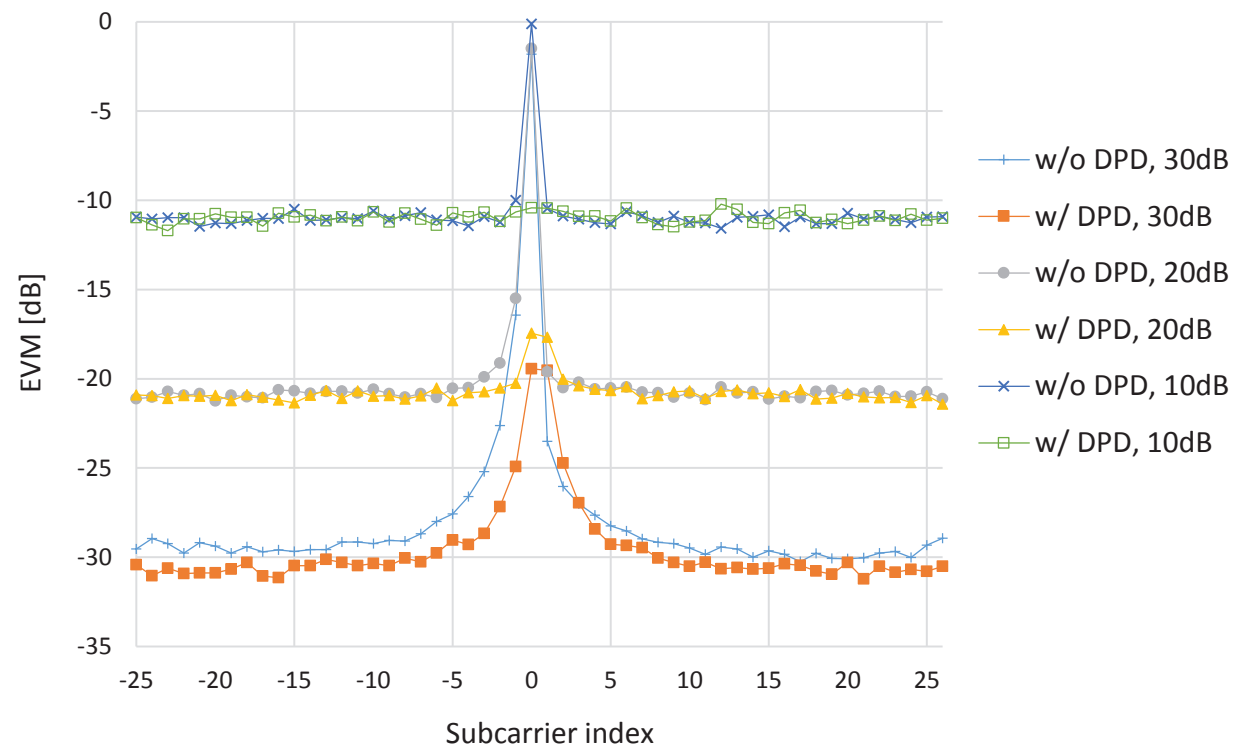

Figure 2. error vector magnitude (EVM) over subcarriers.

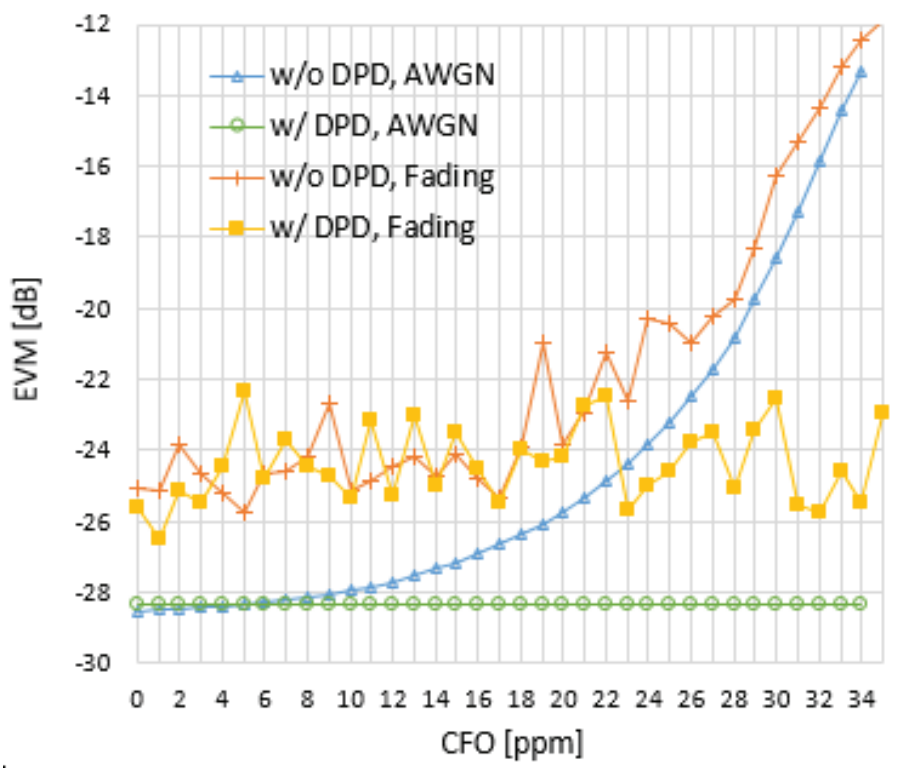

Figure 3. EVM over CFO for channel conditions.

Figure 4 shows the packet error ratio (PER) evaluation results for the proposed digital predistortion scheme compared with results for the conventional design. " $w / D P D$ " refers to the proposed digital predistortion, and " $\mathrm{w} / \mathrm{o}$ DPD" is the conventional design. The simulation is performed with various CFO conditions. As a result, if the proposed digital predistortion scheme was enabled, the PER was significantly improved for the high frequency offset condition, which is higher than $25 \mathrm{ppm}$. This is because of the reduced signal distortion in the low index subcarriers near the DC tone. 


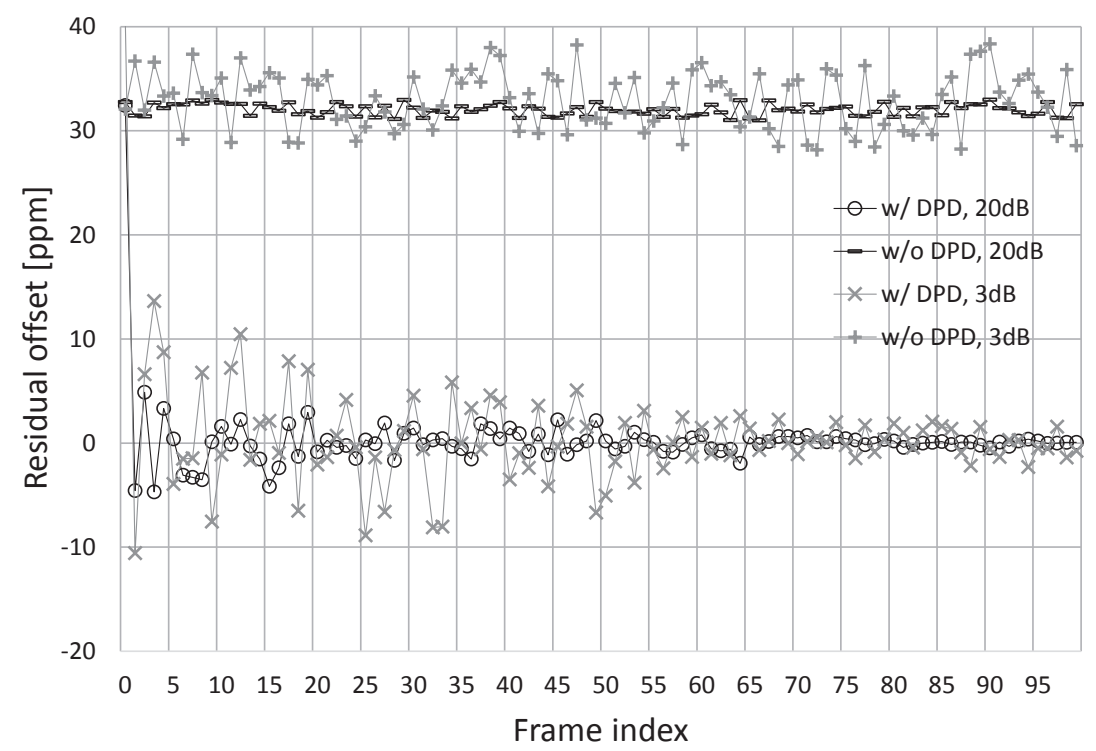

Figure 4. packet error ratio (PER) over CFOs; digital CFO predistortion scheme vs. conventional design.

Figure 5 shows the training evaluation results for the proposed scheme compared with those for the conventional design. The simulation was performed in two average SINR regions: $20 \mathrm{~dB}$ (high SINR) and $3 \mathrm{~dB}$ (low SINR). As a result, if the proposed digital CFO predistortion scheme is adopted, it can reduce the residual CFOs by tracking the offsets.

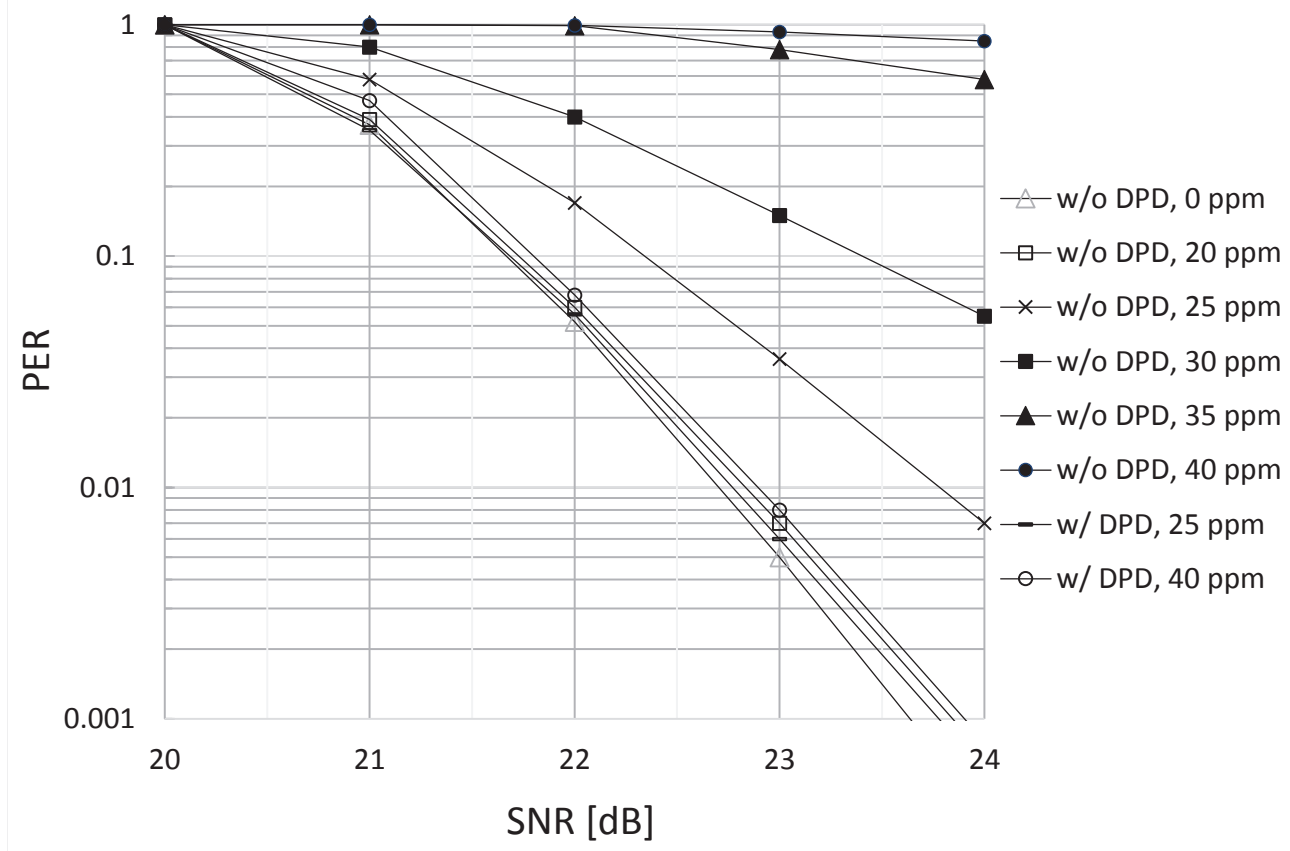

Figure 5. Training of digital predistorted carrier frequency offset; residual offset vs. frame index.

\section{Conclusions}

Wi-Fi enabled IoT devices have been developed to communicate in narrowband in order to take advantage of improved receiver sensitivity, robustness to interference, and energy efficiency. However, in dynamically interfered channels or poor wireless channel conditions, the data rate decreases due to inaccurately estimated CFOs and compensation. If the proposed digital pre-distortion method is applied, such CFO estimation error can be minimized, allowing the above described problem to be 
overcome. Accordingly, data packet can be transmitted at high speed. As a next generation WLAN system that can ensure high efficiency in terms of throughput and coverage, the next version of the IEEE 802.11 WLAN standard, called "high-efficiency WLAN (HEW)", is under discussion [21,22]. IEEE 802.11ax WLANs will evolve toward dynamic bandwidth operation in narrower and wider bandwidths. The proposed digital predistortion scheme will achieve a substantial enhancement in next generation wireless LANs.

Combining effects of CFO and DC offset degrades the signal quality near DC subcarriers in the conventional schemes due to DC cancellers at the receiver side. Futhermore, when the wireless channel is very poor due to noise, interference, and channel effect, it is difficult to accurately estimate the CFO. In this paper, the digital CFO predistortion scheme was proposed for a reliable communication link in dense networks. Simulation-driven evaluation results demonstrated that the proposed scheme can improve received signal quality in terms of packet error rate and error vector magnitude. This work focused on single antenna wireless system targetted for IoT applications. As future work, this work will be extended to combine multiple antenna systems that are more susceptible for carrier frequency offsets and implemented in a real-test bed.

Conflicts of Interest: The author declares no conflict of interest.

\section{References}

1. Connect your life: Wi-Fi and the internet of everything. Wi-Fi Alliance 2014. Available online: http:/ / www. wi-fi.org/system/files/wp_Wi-Fi_Internet_of_Things_Vision_20140110.pdf (accessed on 8 August 2017).

2. IEEE Standard. IEEE Standard for Information technology-Telecommunications and information exchange between systems Local and metropolitan area networks-Specific requirements-Part 11: Wireless LAN Medium Access Control (MAC) and Physical Layer (PHY) Specifications -Amendment 4: Enhancements for Very High Throughput for Operation in Bands below 6 GHz. IEEE Stand. 802.11ac. 2013, doi:10.1109/ieeestd.2014.6744566.

3. IEEE Standard. IEEE Standard for Information technology-Telecommunications and information exchange between systems-Local and metropolitan area networks-Specific requirements-Part 11: Wireless LAN Medium Access Control (MAC) and Physical Layer (PHY) Specifications Amendment 5: Television White Spaces (TVWS) Operation. IEEE Stand. 802.11af. 2014, doi:10.1109/IEEESTD.2014.6744566.

4. IEEE Draft Standard for Information Technology-Telecommunications and Information Exchange Between Systems-Local and Metropolitan Area Networks-Specific Requirements-Part 11: Wireless LAN Medium Access Control (MAC) and Physical Layer (PHY) Specifications: Amendment- Sub 1 GHz License-Exempt Operation. IEEE P802.11ah. 2014, doi:10.1109/ieeestd.2017.7920364.

5. Aust, S.; Prasad, R.V.; Niemegeers, I.G. IEEE 802.11 ah: Advantages in standards and further challenges for sub $1 \mathrm{GHz}$ Wi-Fi. In Proceedings of the 2012 IEEE International Conference on Communications (ICC), Ottawa, ON, Canada, 10-15 June 2012; pp. 6885-6889.

6. Sum, C.S.; Harada, H.; Kojima, F.; Lan, Z.; Funada, R. Smart utility networks in TV white space. IEEE Commun. Mag. 2011, 49, 7.

7. Khorov, E.; Lyakhov, A.; Krotov, A.; Guschin, A. A survey on IEEE 802.11 ah: An enabling networking technology for smart cities. Comput. Commun. 2015, 58, 53-69.

8. Rose, K.; Eldridge, S.; Chapin, L. The Internet of Things (IoT): An Overview-Understanding the Issues and Challenges of a More Connected World. Int. Soc. 2015 Available online: http:/ / www.internetsociety.org/ sites/default/ files/ISOC-IoTOverview-20151022.pdf (accessed on 8 August 2017).

9. Baker, T.; Asim, M.; Tawfik, H.; Aldawsari, B.; Buyya, R. An energy-aware service composition algorithm for multiple cloud-based IoT applications. J. Netw. Comput. Appl. 2017, 89, 96-108.

10. Gubbi, J.; Buyya, R.; Marusic, S.; Palaniswami, M. Internet of Things (IoT): A vision, architectural elements, and future directions. Future Gener. Comput. Syst. 2013, 29, 1645-1660.

11. Ta-Shma, P.; Akbar, A.; Gerson-Golan, G.; Hadash, G.; Carrez, F.; Moessner, K. An ingestion and analytics architecture for iot applied to smart city use cases. IEEE Int. Things J. 2017, doi:10.1109/jiot.2017.2722378.

12. Goth, G. Next-Generation Wi-Fi: As fast as we'll need? IEEE Int. Comp. 2012, 16, 7-9. 
13. Wilhelmsson, L.R.; Lopez, M.M.; Sundman, D. NB-WiFi: IEEE 802.11 and Bluetooth Low Energy Combined for Efficient Support of IoT. In Wireless Communications and Networking Conference (WCNC); IEEE: Piscataway, NJ, USA, 2017; pp. 1-6.

14. Chintalapudi, K.; Radunovic, B.; Balan, V.; Buettener, M.; Yerramalli, S.; Navda, V.; Ramjee, R. WiFi-NC: WiFi over narrow channels. In Proceedings of the 9th USENIX conference on Networked Systems Design and Implementation, Berkeley, CA, USA, 25 April 2012; p. 4.

15. Li, J.; Liu, G.; Giannakis, G.B. Carrier frequency offset estimation for OFDM-based WLANs. IEEE Signal Proc. Lett. 2001, 8, 80-82.

16. D'Amico, A.A.; Marchetti, L.; Morelli, M.; Moretti, M. Frequency estimation in OFDM direct-conversion receivers using a repeated preamble. IEEE Trans. Commun. 2016, 64, 1246-1258.

17. Milos, J.; Polak, L.; Slanina, M. Performance analysis of IEEE 802.11 ac/ax WLAN technologies under the presence of CFO. In Proceedings of the 27th International Conference on Radioelektronika (RADIOELEKTRONIKA), Brno, Czech Republic, 19-20 April 2017; Volume 30, pp. 1-4.

18. Ali, M.A.; Sandhu, M.Y.; Waqar, O.; Rahimo, A.Q. Receiver design for multicarrier transmission systems in presence of Tx/Rx imperfections. Int. J. Commun. Syst. 2017, 30, e3277.

19. Nagai, Y.; Fujimura, A.; Akihara, M.; Nakase, H.; Kameda, S.; Oguma, H.; Tsubouchi, K. A SINR estimation for closed-loop link adaptation of $324 \mathrm{Mbit} / \mathrm{sec}$ WLAN system. In Proceedings of the IEEE 19th International Symposium on Personal, Indoor and Mobile Radio Communications, PIMRC 2008, Cannes, France, 15-18 September 2008; pp. 1-6.

20. Breit, G.H.; Sampath, S.; Vermani, S. TGac Channel Model Addendum. Version 12. IEEE 2010, doi:10.1109/IEEE 802.11-09/0569r0.

21. Cariou, L.; Derham, T.; Rouzic, J.P.L.; Liu, D.; Shao, C.; Xie, F.; Worstell, H.R.; Sampath, H.; Barriac, G.; Jones, V.K.; et al. High-efficiency WLAN. IEEE 2013, 802, 802-811.

22. Nitsche, T.; Cordeiro, C.; Flores, A.B.; Knightly, E.W.; Perahia, E.; Widmer, J.C. High-efficiency WLAN overview. IEEE 2014, 52, 132-141.

(C) 2017 by the author. Licensee MDPI, Basel, Switzerland. This article is an open access article distributed under the terms and conditions of the Creative Commons Attribution (CC BY) license (http://creativecommons.org/licenses/by/4.0/). 